\title{
Wave Breaking for the Modified Two-Component Camassa-Holm System
}

\author{
Wujun Lv and Weiyi Zhu \\ Department of Mathematics, Zhejiang Normal University, Jinhua 321004, China \\ Correspondence should be addressed to Weiyi Zhu; zwy@zjnu.cn
}

Received 8 April 2014; Accepted 21 April 2014; Published 5 May 2014

Academic Editor: Yonghui Xia

Copyright (C) 2014 W. Lv and W. Zhu. This is an open access article distributed under the Creative Commons Attribution License, which permits unrestricted use, distribution, and reproduction in any medium, provided the original work is properly cited.

Some new sufficient conditions to guarantee wave breaking for the modified two-component Camassa-Holm system are established.

\section{Introduction}

This paper concerns the following modified two-component Camassa-Holm system (MCH2, for simplicity):

$$
\begin{gathered}
u_{t}-u_{x x t}+3 u u_{x}-2 u_{x} u_{x x}-u u_{x x x}=-g \rho \bar{\rho}_{x}, \\
t>0, \quad x \in \mathbb{R}, \\
\rho_{t}+(\rho u)_{x}=0, \quad t>0, \quad x \in \mathbb{R}, \\
u(x, t=0)=u_{0}(x), \quad x \in \mathbb{R}, \\
\rho(x, t=0)=\rho_{0}(x), \quad x \in \mathbb{R},
\end{gathered}
$$

where $\rho(x, t)=\left(1-\partial_{x}^{2}\right)\left(\bar{\rho}-\bar{\rho}_{0}\right)(x, t), u(x, t)$ expresses the velocity field, and $g$ is the downward constant acceleration of gravity in applications to shallow water waves. In this paper, we let $g=1$.

Let $\Lambda=\left(1-\partial_{x}^{2}\right)^{(1 / 2)}$; then the operator $\Lambda^{-2}$ can be denoted by its associated Green's function $G=(1 / 2) e^{-|x|}$ as

$$
\left(\Lambda^{-2} f\right)(x)=(G * f)(x)=\frac{1}{2} \int_{\mathbb{R}} e^{-|x-y|} f(y) d y .
$$

Let $\gamma(x, t)=\left(\bar{\rho}-\bar{\rho}_{0}\right)(x, t)$ and $(G * \rho)(x, t)=\gamma(x, t)$. So system (1) is equivalent to the following one:

$$
\begin{gathered}
u_{t}+u u_{x}+\partial_{x} G *\left(u^{2}+\frac{1}{2} u_{x}^{2}+\frac{1}{2} \gamma^{2}-\frac{1}{2} \gamma_{x}^{2}\right)=0, \\
t>0, \quad x \in \mathbb{R}, \\
\gamma_{t}+u \gamma_{x}+G *\left(\left(u_{x} \gamma_{x}\right)_{x}+u_{x} \gamma\right)=0, \quad t>0, \quad x \in \mathbb{R}, \\
u(x, t=0)=u_{0}(x), \quad x \in \mathbb{R}, \\
\gamma(x, t=0)=\gamma_{0}(x), \quad x \in \mathbb{R} .
\end{gathered}
$$

The $\mathrm{MCH} 2$ system admits peaked solutions in the velocity and average density and we refer it to reference [1]. The local posedness, precise blow-up scenarios, and the existence of strong solutions which blow up in finite time can be found in [2-5]. Note that the $\mathrm{MCH} 2$ system is a modified version of the 2-component Camassa-Holm ( $\mathrm{CH} 2$, for simplicity) system to allow a dependence on the average density $\bar{\rho}$ (or depth, in the shallow water interpretation) as well as the pointwise density $\rho$. Meanwhile, the $\mathrm{MCH} 2$ may not be integrable unlike the $\mathrm{CH} 2$ system. The characteristic is that it will amount to strengthening the norm for $\bar{\rho}$ from $L^{2}$ to $H^{1}$ in the potential energy term [5]. Also, the $\mathrm{MCH} 2$ admits the following conserved quantity:

$$
E_{1}=\int_{\mathbb{R}}\left(u^{2}+u_{x}^{2}+\gamma^{2}+\gamma_{x}^{2}\right) d x .
$$


This paper mainly studies wave breaking phenomenon, and we aim at improving previous results which were proved in $[3,6]$. Our method is partially motivated by [7]. The remaining of this paper is organized as follows. In Section 2, we introduce some preliminaries. In Section 3, we establish a new blow-up criterion for the $\mathrm{MCH} 2$. Finally, we establish a similar criterion for the $\mathrm{CH} 2$ system in Section 4.

\section{Preliminaries}

In this section, we recall some results without the proofs for conciseness. The first one is concerning local well-posedness and blow-up scenario.

Lemma 1 (see [2]). Given $X_{0}=\left(u_{0}, \gamma_{0}\right)^{T} \in H^{s} \times H^{s}$ to system (3), $s \geq 3 / 2$, there exists a maximal $T=T\left(\left\|X_{0}\right\|_{H^{s} \times H^{s}}\right)>0$, and a unique solution $X=(u, \gamma)^{T} \in H^{s} \times H^{s}$ to system (3). Then the corresponding solutions blow up in finite time if and only if

$$
\lim _{t \rightarrow T} \inf _{x \in \mathbb{R}}\left\{u_{x}(x, t)\right\}=-\infty \quad \text { or } \quad \liminf _{t \rightarrow T} \inf _{x \in \mathbb{R}}\left\{\gamma_{x}(x, t)\right\}=-\infty .
$$

We also need to introduce the standard particle trajectory [8]. Let $q(x, t)$ be the particle line evolved by the solution; that is, it satisfies

$$
\begin{gathered}
q_{t}=u(q, t), \quad 0<t<T, x \in \mathbb{R}, \\
q(x, 0)=x, \quad x \in \mathbb{R} .
\end{gathered}
$$

Taking the derivative with respect to $x$, we get

$$
\frac{d q_{t}}{d x}=q_{x t}=u_{x}(q, t) q_{x}, \quad t \in(0, T) .
$$

Hence

$$
q_{x}(x, t)=\exp \left\{\int_{0}^{t} u_{x}(q, s) d s\right\}, \quad q_{x}(x, 0)=1 .
$$

Thus, the map $q(\cdot, t)$ is a diffeomorphism of the real line.

\section{Blowup for the MCH2 System}

In this section, we establish a new sufficient condition to guarantee blowup for system (3), which is an improvement of that in [3].

Theorem 2. Suppose $X_{0}=\left(u_{0}, \gamma_{0}\right)^{T} \in H^{s} \times H^{s}$ to system (3), $s>3 / 2$ and $\rho_{0}\left(x_{0}\right)=0$. And the initial data satisfies the following two conditions:

$$
\begin{gathered}
\text { (i) } \rho_{0}\left(x_{0}\right) \geq 0 \quad \text { on }\left(-\infty, x_{0}\right), \\
\rho_{0}\left(x_{0}\right) \leq 0 \quad \text { on }\left(x_{0}, \infty\right), \\
\text { (ii) } u_{0}^{\prime}\left(x_{0}\right)<-\left|u_{0}\left(x_{0}\right)\right|,
\end{gathered}
$$

for some point $x_{0} \in \mathbb{R}$. Then the solution $X=(u, \gamma)^{T}$ to our system (3) with initial value $X_{0}$ blows up in finite time.
Remark 3. In [17] conditions $\int_{-\infty}^{x_{0}} e^{\xi} y_{0}(\xi) d \xi \geq 0$ and $\int_{x_{0}}^{\infty} e^{-\xi} y_{0}(\xi) d \xi \leq 0$ are needed to guarantee blowup, which implies condition (10). In addition, $y_{0}\left(x_{0}\right)=0$ is required. So obviously Theorem 2 is an improvement of that in [3]. On the other hand, our condition is a local version and is easy to check. For nonlocal conditions, we refer to $[5,9]$.

\section{Now we give a proof for Theorem 2 .}

Proof. Let us first consider the case $X_{0}=\left(u_{0}, \gamma_{0}\right)^{T} \in H^{2} \times$ $H^{2}$. As in [10], we will look for $(d / d t) u_{x}(q(x, t), t)$. Applying $\partial_{x}^{2}(G * f)=G * f-f$ to differentiate (3) with respect to $x$ yields

$$
\begin{aligned}
u_{t x}+u u_{x x}= & -\frac{1}{2} u_{x}^{2}+u^{2}+\frac{1}{2} \gamma^{2} \\
& -\frac{1}{2} \gamma_{x}^{2}-G *\left(\frac{1}{2} u_{x}^{2}+u^{2}+\frac{1}{2} \gamma^{2}-\frac{1}{2} \gamma_{x}^{2}\right)
\end{aligned}
$$

Let $0<T<T^{*}$. Recalling that $u \in C^{1}\left([0, T), H^{2}\right)$, we show that $u$ and $u_{x}$ are continuous on $[0, T) \times \mathbb{R}$ and $x \rightarrow$ $u(t, x)$ is Lipschitz, uniformly with respect to $t$ in any compact time interval in $[0, T)$.

We get

$$
\begin{aligned}
\frac{d}{d t} u_{x}\left(q\left(x_{0}, t\right), t\right) & \\
= & \left(u_{t x}+u u_{x x}\right)\left(q\left(x_{0}, t\right), t\right) \\
= & \left(-\frac{1}{2} u_{x}^{2}+u^{2}+\frac{1}{2} \gamma^{2}-\frac{1}{2} \gamma_{x}^{2}\right)\left(t, q\left(t, x_{0}\right)\right) \\
& -G *\left(\frac{1}{2} u_{x}^{2}+u^{2}+\frac{1}{2} \gamma^{2}-\frac{1}{2} \gamma_{x}^{2}\right) \\
\leq & -\frac{1}{2} u_{x}^{2}+\frac{1}{2} u^{2}
\end{aligned}
$$

where we used $G *\left(u^{2}+(1 / 2) u_{x}^{2}\right) \geq(1 / 2) u^{2}, \gamma_{x}^{2}(x, t)-\gamma^{2}(x, t) \leq$ $\gamma_{x}^{2}\left(q\left(x_{0}, t\right), t\right)-\gamma^{2}\left(q\left(x_{0}, t\right), t\right)$, and $\rho\left(q\left(x_{0}, t\right), t\right)=0$.

As

$$
\frac{d}{d t} \rho(q(x, t), t) q_{x}(x, t)=0
$$

we get

$$
\rho\left(q\left(x_{0}, t\right), t\right) q_{x}\left(x_{0}, t\right)=\rho_{0}\left(x_{0}\right)=0
$$

it is easy to get $q_{x}\left(x_{0}, t\right)>0$ in $(8)$, so $\rho\left(q\left(x_{0}, t\right), t\right)=0$.

Consider $\gamma_{x}^{2}(x, t)-\gamma^{2}(x, t) \leq \gamma_{x}^{2}\left(q\left(x_{0}, t\right), t\right)-\gamma^{2}\left(q\left(x_{0}, t\right), t\right)$; we can refer to [3].

The obvious factorization $u^{2}-u_{x}^{2}=\left(u-u_{x}\right)\left(u+u_{x}\right)$; this leads us to study the functions of the form:

$$
\begin{gathered}
I\left(x_{0}, t\right)=e^{q\left(x_{0}, t\right)}\left(u-u_{x}\right)\left(q\left(x_{0}, t\right), t\right), \\
I I\left(x_{0}, t\right)=e^{-q\left(x_{0}, t\right)}\left(u+u_{x}\right)\left(q\left(x_{0}, t\right), t\right) .
\end{gathered}
$$


Computing the derivatives with respect to $t$ using the definition of the flow map (6) gives

$$
\begin{aligned}
& I_{t}\left(x_{0}, t\right)=e^{q\left(x_{0}, t\right)}\left[u^{2}-u u_{x}+\left(u_{t}+u u_{x}\right)\right. \\
&\left.-\left(u_{x t}+u u_{x x}\right)\right]\left(q\left(x_{0}, t\right), t\right) \\
&= e^{q\left(x_{0}, t\right)}\left[-u u_{x}+\frac{1}{2} u_{x}^{2}-\frac{1}{2}\left(\gamma^{2}-\gamma_{x}^{2}\right)+\left(G-\partial_{x} G\right)\right. \\
&\left.*\left(u^{2}+\frac{1}{2} u_{x}^{2}+\frac{1}{2}\left(\gamma^{2}-\gamma_{x}^{2}\right)\right)\right] \\
& \geq e^{q\left(x_{0}, t\right)}\left(\frac{1}{2} u^{2}-u u_{x}+\frac{1}{2} u_{x}^{2}\right) \\
&= \frac{1}{2} e^{q\left(x_{0}, t\right)}\left(u-u_{x}\right)^{2} \geq 0
\end{aligned}
$$

In fact, the next lemma will be used.

Lemma 4. Consider

$$
\left(G \pm \partial_{x} G\right) *\left(u^{2}+\frac{1}{2} u_{x}^{2}\right) \geq \frac{1}{2} u^{2}
$$

Proof. Consider

$$
\begin{aligned}
& \frac{1}{2} e^{-x} \int_{-\infty}^{x} e^{\xi}\left(u^{2}+u_{x}^{2}\right)(\xi) d \xi \\
& \quad \geq e^{-x} \int_{-\infty}^{x} e^{\xi} u u_{x} d \xi=\frac{1}{2} u^{2}(x)-\frac{1}{2} e^{-x} \int_{-\infty}^{x} e^{\xi} u^{2}(\xi) d \xi
\end{aligned}
$$

So we get

$$
\frac{1}{2} e^{-x} \int_{-\infty}^{x} e^{\xi}\left(u^{2}+\frac{1}{2} u_{x}^{2}\right)(\xi) d \xi \geq \frac{1}{4} u^{2}
$$

The same computations also obtain that

$$
\frac{1}{2} e^{x} \int_{-\infty}^{x} e^{-\xi}\left(u^{2}+\frac{1}{2} u_{x}^{2}\right)(\xi) d \xi \geq \frac{1}{4} u^{2}
$$

We have

$$
\begin{aligned}
& \left(G-\partial_{x} G\right)=e^{-x} \int_{-\infty}^{x} e^{\xi}\left(u^{2}+\frac{1}{2} u_{x}^{2}\right)(\xi) d \xi \\
& \left(G+\partial_{x} G\right)=\frac{1}{2} e^{x} \int_{-\infty}^{x} e^{-\xi}\left(u^{2}+\frac{1}{2} u_{x}^{2}\right)(\xi) d \xi
\end{aligned}
$$

taking the linear combination in the two last inequalities implies estimate (17).

Similarly,

$$
I I_{t}\left(x_{0}, t\right)=-\frac{1}{2} e^{-q\left(x_{0}, t\right)}\left(u+u_{x}\right)^{2} \leq 0 .
$$

It is convenient to establish the following fundamental proposition.
Proposition 5. $u$ as in Theorem 2. Set

$$
\begin{aligned}
& I\left(x_{0}, t\right)=e^{q\left(x_{0}, t\right)}\left(u-u_{x}\right)\left(q\left(x_{0}, t\right), t\right), \\
& I I\left(x_{0}, t\right)=e^{-q\left(x_{0}, t\right)}\left(u+u_{x}\right)\left(q\left(x_{0}, t\right), t\right) .
\end{aligned}
$$

Then, for all $x \in \mathbb{R}$, the function $t \rightarrow I\left(x_{0}, t\right)$ is monotonically increasing and $t \rightarrow I I\left(t, x_{0}\right)$ is monotonically decreasing.

It is easy to factorize

$$
\left(u^{2}-u_{x}^{2}\right)\left(q\left(x_{0}, t\right), t\right)=I\left(x_{0}, t\right) I I\left(x_{0}, t\right) ;
$$

from inequality (12) we get

$$
\frac{d}{d t} u_{x}\left(q\left(x_{0}, t\right), t\right) \leq \frac{1}{2} I\left(x_{0}, t\right) I I\left(x_{0}, t\right)
$$

Now let $x_{0}$ be such that $u_{0}^{\prime}\left(x_{0}\right)<-\left|u_{0}\left(x_{0}\right)\right|$. Proposition 5 yields, for all $t \in[0, T)$,

$$
I\left(x_{0}, t\right) \geq I_{0}\left(x_{0}\right)>0, \quad I I\left(x_{0}, t\right) \leq I_{0}\left(x_{0}\right)<0,
$$

where we used $u_{0}^{\prime}\left(x_{0}\right)<-\left|u_{0}\left(x_{0}\right)\right|$, then we get $I_{0}\left(x_{0}\right)>0$ and $I I_{0}\left(x_{0}\right)<0$.

Assume, by contradiction, $T=\infty$; set $A(t)=$ $u_{x}\left(q\left(x_{0}, t\right), t\right)$; thus we get

$$
A^{\prime}(t) \leq \frac{1}{2} I\left(x_{0}, t\right) I I\left(x_{0}, t\right) \leq \frac{1}{2} I_{0}\left(x_{0}\right) I I_{0}\left(x_{0}\right)<0 .
$$

Set $\beta_{0}=(1 / 2)\left(u_{0}^{\prime 2}-u_{0}^{2}\right)\left(x_{0}\right)$; then $A(t) \leq A(0)-\beta_{0} t$; we can find $t_{0}$ such that $\left(A(0)-\beta_{0} t_{0}\right)^{2} \geq E_{1}\left(E_{1}=\|u(t)+\gamma(t)\|_{H^{1}}^{2}=\right.$ $\left.\left\|u_{0}+\gamma_{0}\right\|_{H^{1}}^{2}\right)$. For $t \geq t_{0}$, then $A(t) \leq A\left(t_{0}\right)$; we obtain

$$
\begin{aligned}
A^{\prime}(t) \leq \frac{1}{2} I\left(x_{0}, t\right) I I\left(x_{0}, t\right) & =\frac{1}{2}\left(u^{2}-u_{x}^{2}\right)\left(q\left(x_{0}, t\right), t\right) \\
& \leq \frac{1}{2}\left(\frac{1}{2} E_{1}-A(t)^{2}\right) \\
& \leq-\frac{1}{4} A(t)^{2} .
\end{aligned}
$$

This implies that, for $t \geq t_{0}$,

$$
A(t) \leq \frac{4 A\left(t_{0}\right)}{4-\left(t-t_{0}\right) A\left(t_{0}\right)} .
$$

From above, $u_{x}\left(q\left(x_{0}, t\right), t\right)$ must blow up in finite time, and $T^{*}=t_{0}+4 / A\left(t_{0}\right)<\infty$, so the condition of the blowup scenario (5) is fulfilled.

\section{Blowup for the $\mathrm{CH} 2$ System}

In this section, we consider the following two-component Camassa-Holm system:

$$
\begin{gathered}
u_{t}+u u_{x}+\partial_{x}\left(G *\left(u^{2}+\frac{1}{2} u_{x}^{2}+\frac{\delta}{2} \rho^{2}\right)\right)=0 \\
t>0, \quad x \in \mathbb{R}, \\
\rho_{t}+(\rho u)_{x}=0, \quad t>0, \quad x \in \mathbb{R} .
\end{gathered}
$$


The $\mathrm{CH} 2$ system appears initially in [11]. Wave breaking mechanism was discussed in $[3,12-14]$. The existence of global solutions was analyzed in $[6,15,16]$. This system also has the following conservation laws [17]:

$$
\begin{aligned}
& E_{1}=\int_{\mathbb{R}}\left(u^{2}+u_{x}^{2}+\delta \rho^{2}\right) d x, \\
& E_{2}=\int_{\mathbb{R}}\left(u^{3}+u u_{x}^{2}+\delta u \rho^{2}\right) d x .
\end{aligned}
$$

In [6], a blow-up condition is established as $y_{0}\left(x_{0}\right)=0$, $\int_{-\infty}^{x_{0}} e^{\xi} y_{0}(\xi) d \xi \geq 0$ and $\int_{x_{0}}^{\infty} e^{-\xi} y_{0}(\xi) d \xi \leq 0$; here $y_{0}\left(x_{0}\right)=$ $\left(1-\partial_{x}^{2}\right) u_{0}\left(x_{0}\right)$. Similar to Theorem 2 , we can do the following improvement.

Theorem 6. Suppose $X_{0}=\left(u_{0}, \rho_{0}\right)^{T} \in H^{s} \times H^{s-1}$ to system (30), $s \geq 3 / 2$, and $\rho\left(x_{0}\right)=0$; furthermore

$$
u_{0}^{\prime}\left(x_{0}\right)<-\left|u_{0}\left(x_{0}\right)\right|,
$$

for some point $x_{0} \in \mathbb{R}$. Then the solution to our system (30) with initial value $X_{0}$ blows up in finite time.

The proof is similar to Theorem 2 and we omit it.

\section{Conflict of Interests}

The authors declare that there is no conflict of interests regarding the publication of this paper.

\section{Acknowledgment}

The authors thank anonymous reviewers for their valuable comments and careful reading.

\section{References}

[1] D. D. Holm, L. Ó. Náraigh, and C. Tronci, "Singular solutions of a modified two-component Camassa-Holm equation," Physical Review E-Statistical, Nonlinear, and Soft Matter Physics, vol. 79, no. 1, Article ID 016601, 13 pages, 2009.

[2] C. Guan, K. H. Karlsen, and Z. Yin, "Well-posedness and blow-up phenomena for a modified two-component CamassaHolm equation," in Proceedings of the 2008-2009 Special Year in Nonlinear Partial Differential Equations, pp. 199-220, Contemporary Mathematics. American Mathematical Society, 2010.

[3] Z. Guo and M. Zhu, "Wave breaking for a modified twocomponent Camassa-Holm system," Journal of Differential Equations, vol. 252, no. 3, pp. 2759-2770, 2012.

[4] L. Jin and Z. Guo, "A note on a modified two-component Camassa-Holm system," Nonlinear Analysis: Real World Applications, vol. 13, no. 2, pp. 887-892, 2012.

[5] Z. Guo, M. Zhu, and L. Ni, "Blow-up criteria of solutions to a modified two-component CamassaHolm system," Nonlinear Analysis: Real World Applications, vol. 12, no. 6, pp. 3531-3540, 2011.

[6] Z. Guo, "Blow-up and global solutions to a new integrable model with two components," Journal of Mathematical Analysis and Applications, vol. 372, no. 1, pp. 316-327, 2010.
[7] L. Brandolese, "Local-in-space criteria for blowup in shallow water and dispersive rod equations," Communications in Mathematical Physics, 2014.

[8] Y. Zhou, "Wave breaking for a shallow water equation," Nonlinear Analysis: Theory, Methods and Applications, vol. 57, no. 1, pp. 137-152, 2004.

[9] W. Lv, A. Alsaedi, T. Hayat, and Y. Zhou, "Wave breaking and infinite propagation speed for a modified two-component Camassa-Holm system with $\kappa \neq 0$," Journal of Inequalities and Applications, no. 1, article 125, 2014.

[10] A. Constantin, "Existence of permanent and breaking waves for a shallow water equation: a geometric approach," Annales de l'Institut Fourier, vol. 50, no. 2, pp. 321-362, 2000.

[11] P. J. Olver and P. Rosenau, "Tri-Hamiltonian duality between solitons and solitary-wave solutions having compact support," Physical Review E-Statistical Physics, Plasmas, Fluids, and Related Interdisciplinary Topics, vol. 53, no. 2, pp. 1900-1906, 1996.

[12] Z. Guo, "Asymptotic profiles of solutions to the two-component Camassa-Holm system," Nonlinear Analysis: Theory, Methods and Applications, vol. 75, no. 1, pp. 1-6, 2012.

[13] Z. Guo and L. Ni, "Persistence properties and unique continuation of solutions to a two-component Camassa-Holm equation," Mathematical Physics Analysis and Geometry, vol. 14, no. 2, pp. 101-114, 2011.

[14] Z. Guo and M. Zhu, "Wave breaking and measure of momentum support for an integrable Camassa-Holm system with two components," Studies in Applied Mathematics, vol. 130, pp. 417430, 2013.

[15] G. Gui and Y. Liu, "On the global existence and wave-breaking criteria for the two-component Camassa-Holm system," Journal of Functional Analysis, vol. 258, no. 12, pp. 4251-4278, 2010.

[16] Z. Guo and Y. Zhou, "On solutions to a two-component generalized Camassa-Holm equation," Studies in Applied Mathematics, vol. 124, no. 3, pp. 307-322, 2010.

[17] A. Constantin and R. I. Ivanov, "On an integrable twocomponent Camassa-Holm shallow water system," Physics Letters A: General, Atomic and Solid State Physics, vol. 372, no. 48, pp. 7129-7132, 2008. 


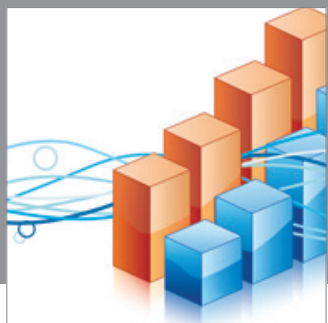

Advances in

Operations Research

mansans

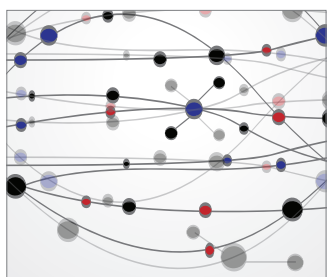

The Scientific World Journal
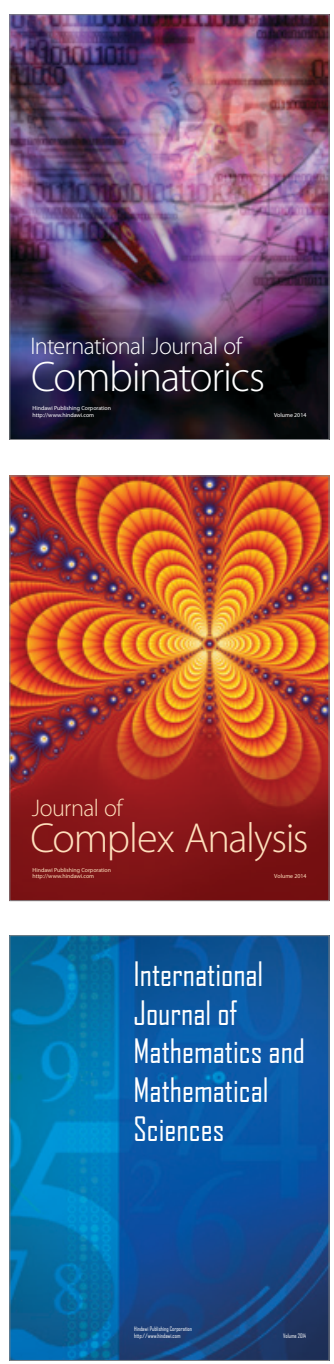
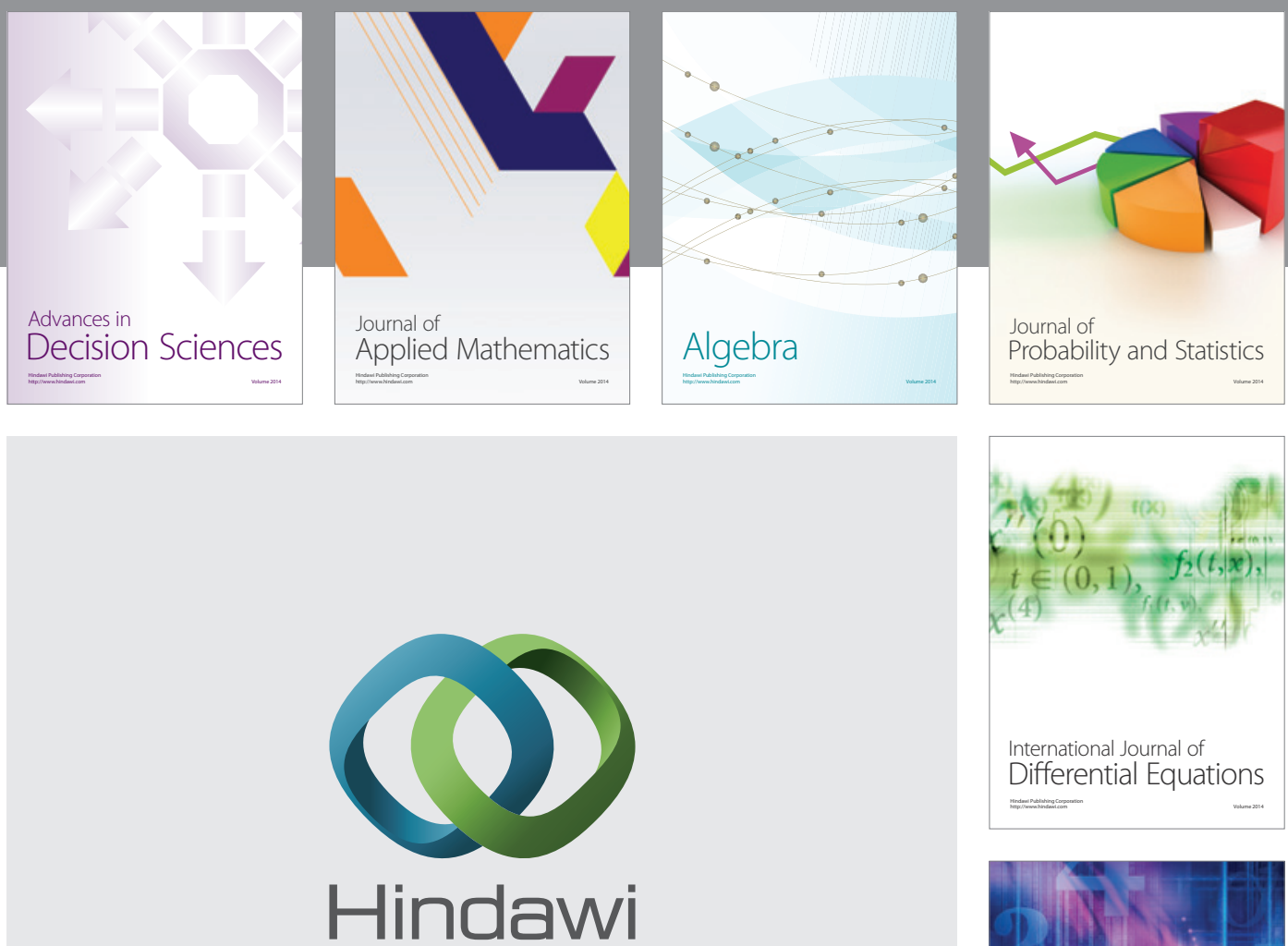

Submit your manuscripts at http://www.hindawi.com
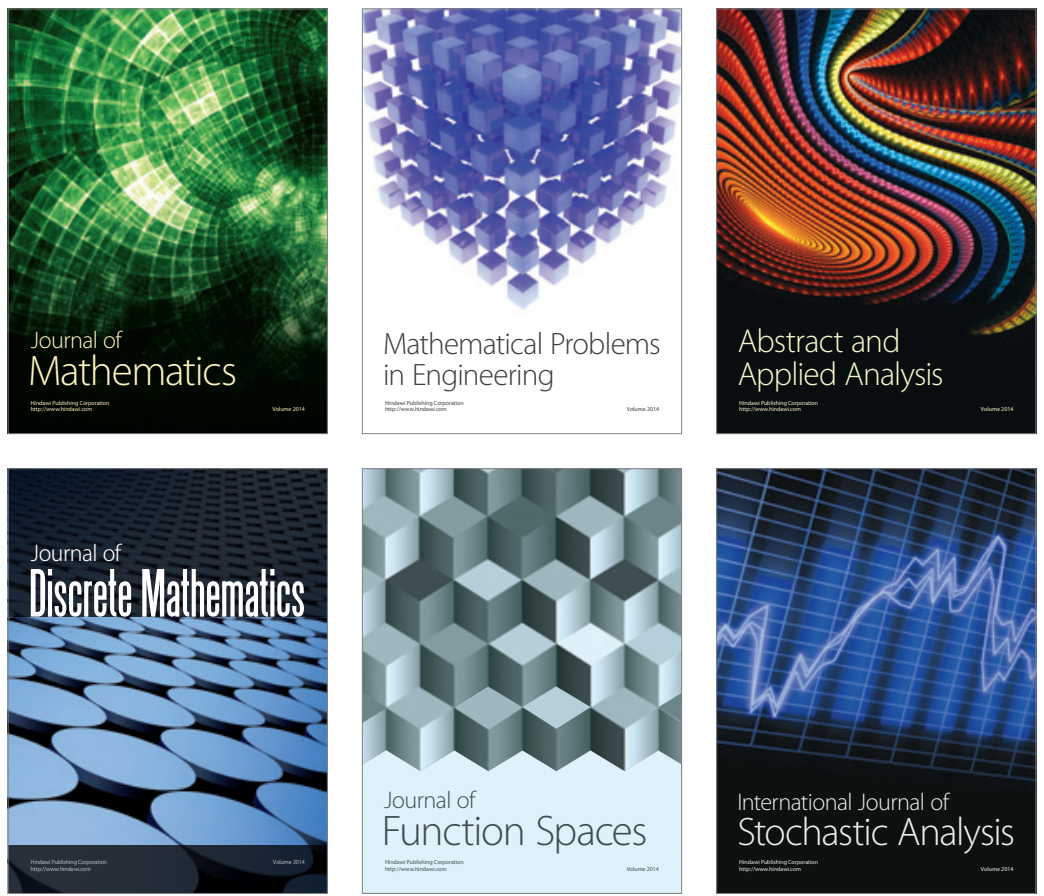

Journal of

Function Spaces

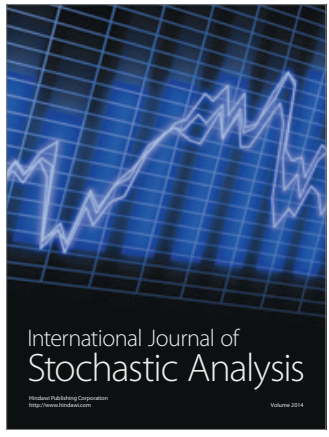

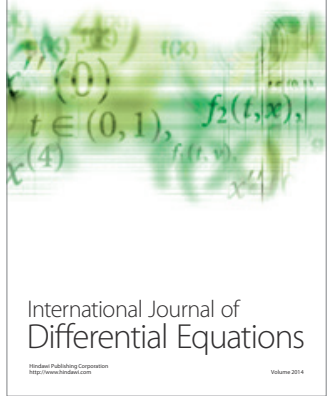
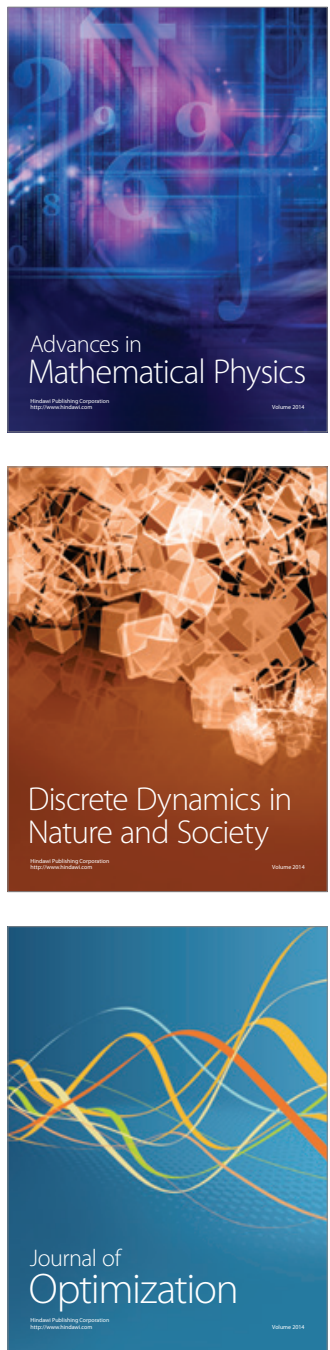